\title{
Phylogenetic Analysis of Ruminococcus flavefaciens, the Type Species of the Genus Ruminococcus, Does Not Support the Reclassification of Streptococcus hansenii and Peptostreptococcus productus as Ruminococci
}

\author{
ANNE WILLEMS* AND MATTHEW D. COLLINS \\ Department of Microbiology, Institute of Food Research, Reading Laboratory, \\ Earley Gate, Reading RG6 2EF, United Kingdom
}

\begin{abstract}
The 16S rRNA gene sequence of the type strain of Ruminococcus flavefaciens, the type species of the genus Ruminococcus, was determined by PCR direct sequencing. A comparative sequence analysis showed that $R$. flavefaciens is phylogenetically related to a small cluster (cluster IV of Collins et al. [M. D. Collins, P. A. Lawson, A. Willems, J. J. Cordoba, J. Fernandez-Garayzabal, P. Garcia, J. Cai, H. Hippe, and J. A. E. Farrow, Int. J. Syst. Bacteriol. 44:812-826, 1994]) of organisms which includes several Clostridium and Eubacterium species. $R$. flavefaciens was found to be phylogenetically only remotely related to Ruminococcus gnavus, Ruminococcus torques, Peptostreptococcus productus, and Streptococcus hansenii. These findings demonstrate that the genus Ruminococcus is not a monophyletic group, and the proposed transfer of $P$. productus and $S$. hansenii to this genus (T. Ezaki, N. Li, Y. Hashimoto, H. Miura, and H. Yamamoto, Int. J. Syst. Bacteriol. 44:130-136, 1994) is not supported.
\end{abstract}

The taxonomic positions of Peptostreptococcus productus (Prévot 1941) Smith 1957 and Streptococcus hansenii (Holdeman and Moore 1974) have always been uncertain $(5,7,12) . P$. productus is saccharolytic and differs markedly in this respect from other peptostreptococci, which are asaccharolytic and use peptones and amino acids as their energy sources. $P$. productus also differs from other peptostreptococci in containing a mesodiaminopimelic acid directly cross-linked murein (type A1 $\gamma$ ) and in having a significantly higher DNA G $+C$ content $(4,8)$. $S$. hansenii closely resembles $P$. productus (e.g., in saccharolytic behavior) but differs from most authentic streptococcal species in being obligately anaerobic and in having a type A1 $y$ murein (12). Ezaki et al. $(4,11)$, using $16 \mathrm{~S}$ rRNA gene sequencing, demonstrated that $P$. productus and $S$. hansenii are phylogenetically closely related to Ruminococcus gnavus, Ruminococcus torques, Coprococcus eutactus, and Clostridium coccoides. On the basis of these findings and phenotypic evidence, Ezaki et al. proposed that $P$. productus and $S$. hansenii should be reclassified in the genus Ruminococcus (4) as Ruminococcus productus and Ruminococcus hansenii, respectively. In a more comprehensive analysis of low-G+C-content gram-positive bacteria (2), $P$. productus and $S$. hansenii were found to be members of a large cluster (cluster XIVa) which included 14 clostridial species, "Acetitomaculum ruminis," Coprococcus eutactus, Eubacterium cellulosolvens, and Roseburia cecicola. Furthermore, the closest relative of $P$. productus and $S$. hansenii was clearly Clostridium coccoides, not $R$. torques (2). In this context it is pertinent to note the suggestion of several authors $(1,8)$ that the ovoid fermentative ruminococcal species $(R u$ minococcus callidus, $R$. gnavus, Ruminococcus lactaris, Ruminococcus obeum, and $R$. torques) may represent a separate

\footnotetext{
* Corresponding author. Mailing address: Department of Microbiology, Institute of Food Research, Reading Laboratory, Earley Gate, Whiteknights Road, Reading RG6 2EF, United Kingdom.
}

genus distinct from the spherical ruminococci Ruminococcus albus and Ruminococcus bromii and the type species of the genus Ruminococcus, Ruminococcus flavefaciens. Thus, the genus Ruminococcus may be heterogeneous, and a close phylogenetic relationship to $R$. gnavus and $R$. torques does not necessarily imply a close affinity with $R$. flavefaciens. To establish the validity of transferring $P$. productus and $S$. hansenii to the genus Ruminococcus, we determined the 16S rRNA gene sequence of $R$. flavefaciens, and in this paper we describe the results of a phylogenetic analysis.

The almost complete 16S rRNA gene sequence of the type strain of $R$. flavefaciens (NCFB 2213 [= ATCC 19208]) was determined by using PCR direct DNA sequencing. Lyophilized cells were resuspended in $500 \mu$ l of TES buffer $(0.05 \mathrm{M}$ Tris-

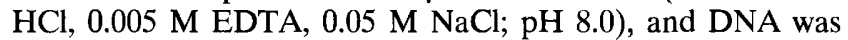
extracted and purified as described previously (10). A large fragment of the $16 \mathrm{~S}$ rRNA gene was amplified by PCR by using conserved primers (5' GAGAGTTTGATCCTGGCTCAGGA and 5' AAGGAGGTGATCCAGCCGCA) proximal to the 5' and $3^{\prime}$ ends of the gene as described by Hutson et al. (9). The fragment was purified by using a Prep-A-Gene kit (Bio-Rad, Hercules, Calif.) according to the manufacturer's instructions and was sequenced by using a Taq DyeDeoxy Terminator Cycle Sequencing Kit (Applied Biosystems, Inc., Foster City, Calif.) and a model 373A automatic sequencer (Applied Biosystems).

The sequence which we determined consisted of 1,448 nucleotides. Identical sequences were obtained for the type strains of $R$. flavefaciens obtained from different culture collections (NCFB 2213 and ATCC 19208). Database searches indicated that $R$. flavefaciens was a member of the Clostridium subphylum of the gram-positive bacteria. The highest levels of sequence relatedness were observed with Clostridium leptum (level of similarity, approximately $88.3 \%$ ) and other members of Clostridium cluster IV (range of levels of similarity, approx- 
TABLE 1. Similarity values for the $16 \mathrm{~S}$ rRNA genes of ruminococcal species and representatives of Clostridium clusters IV and XIVa

\begin{tabular}{|c|c|c|c|c|c|c|c|c|c|c|c|c|c|c|c|c|c|c|c|c|c|}
\hline \multirow[b]{3}{*}{ Species } & \multicolumn{21}{|c|}{$\%$ Similarity to: } \\
\hline & \multicolumn{7}{|c|}{ Cluster IV organisms } & \multicolumn{14}{|c|}{ Cluster XIVa organisms } \\
\hline & 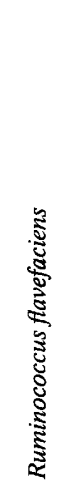 & 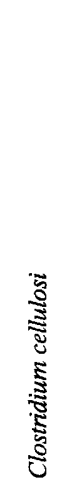 & 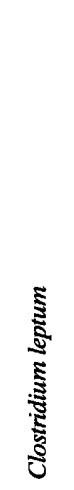 & 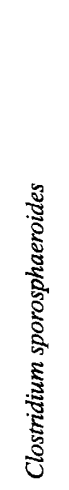 & 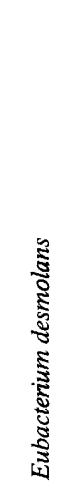 & 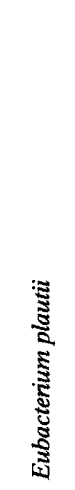 & 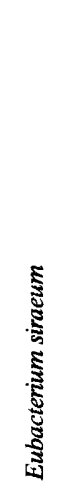 & 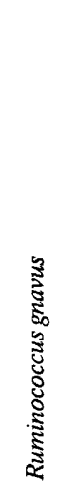 & 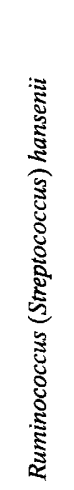 & 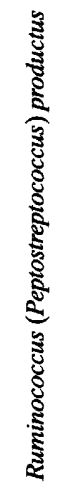 & 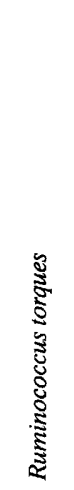 & 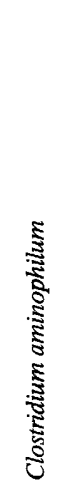 & 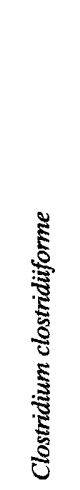 & 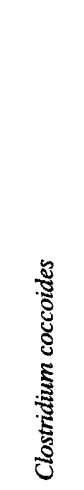 & 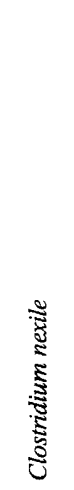 & 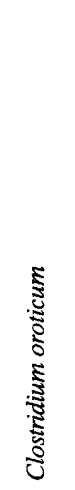 & 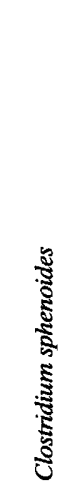 & 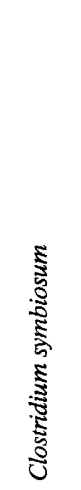 & 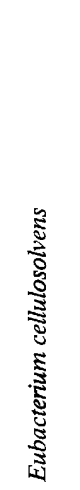 & 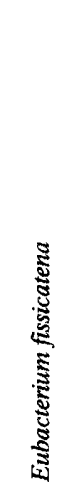 & 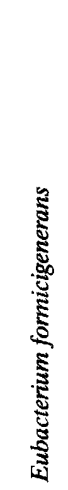 \\
\hline Clostridium cellulosi & 87.5 & & & & & & & & & & & & & & & & & & & & \\
\hline Clostridium leptum & 88.3 & 88.3 & & & & & & & & & & & & & & & & & & & \\
\hline Clostridium sporosphaeroides & 86.2 & 88.7 & 93.4 & & & & & & & & & & & & & & & & & & \\
\hline Eubacterium desmolans & 87.0 & 97.2 & 87.4 & 86.4 & & & & & & & & & & & & & & & & & \\
\hline Eubacterium plautii & 85.8 & 85.4 & 87.7 & 86.7 & 88.9 & & & & & & & & & & & & & & & & \\
\hline Eubacterium siraeum & 88.5 & 86.8 & 86.6 & 85.7 & 86.7 & 86.8 & & & & & & & & & & & & & & & \\
\hline Ruminococcus gnavus & 79.0 & 76.5 & 78.5 & 77.3 & 79.8 & 79.0 & 80.0 & & & & & & & & & & & & & & \\
\hline Ruminococcus (Streptococcus) hansenii & 83.9 & 80.2 & 82.5 & 80.7 & 82.6 & 82.9 & 83.6 & 86.5 & & & & & & & & & & & & & \\
\hline Ruminococcus (Peptostreptococcus) productus & 83.1 & 80.0 & 82.3 & 80.3 & 82.6 & 82.5 & 82.2 & 84.7 & 93.0 & & & & & & & & & & & & \\
\hline Ruminococcus torques & 82.6 & 80.0 & 81.8 & 80.8 & 82.2 & 82.8 & 82.8 & 87.6 & 91.1 & 90.3 & & & & & & & & & & & \\
\hline Clostridium aminophilum & 83.0 & 81.0 & 83.6 & 82.0 & 82.9 & 83.1 & 82.8 & 84.4 & 90.3 & 88.8 & 89.2 & & & & & & & & & & \\
\hline Clostridium clostridiiforme & 82.3 & 79.9 & 83.1 & 81.2 & 82.5 & 83.0 & 82.3 & 87.1 & 92.9 & 90.9 & 92.0 & 91.0 & & & & & & & & & \\
\hline Clostridium coccoides & 84.6 & 81.1 & 83.6 & 81.6 & 84.1 & 84.6 & 83.6 & 88.0 & 95.6 & 96.1 & 92.9 & 91.0 & 93.6 & & & & & & & & \\
\hline Clostridium nexile & 83.3 & 80.2 & 82.6 & 81.6 & 82.9 & 82.5 & 83.0 & 88.7 & 91.7 & 89.8 & 94.2 & 89.6 & 92.9 & 92.2 & & & & & & & \\
\hline Clostridium oroticum & 84.3 & 80.6 & 83.2 & 81.9 & 83.0 & 84.0 & 83.2 & 88.4 & 91.7 & 90.8 & 95.0 & 89.9 & 93.8 & 93.5 & 93.0 & & & & & & \\
\hline Clostridium sphenoides & 83.3 & 80.7 & 83.7 & 82.8 & 83.4 & 84.1 & 84.0 & 86.6 & 91.8 & 90.2 & 92.2 & 91.4 & 93.4 & 93.3 & 92.3 & 93.0 & & & & & \\
\hline Clostridium symbiosum & 83.3 & 80.9 & 82.7 & 80.9 & 82.3 & 82.9 & 83.6 & 85.2 & 91.6 & 89.7 & 91.8 & 92.6 & 93.5 & 91.9 & 91.9 & 93.4 & 92.9 & & & & \\
\hline Eubacterium cellulosolvens & 81.2 & 79.4 & 82.0 & 80.7 & 81.8 & 81.7 & 82.2 & 84.8 & 91.5 & 89.4 & 89.4 & 90.7 & 91.4 & 91.9 & 90.1 & 89.8 & 90.6 & 90.3 & & & \\
\hline Eubacterium fissicatena & 84.0 & 80.5 & 82.8 & 81.5 & 82.6 & 83.6 & 82.9 & 88.9 & 91.7 & 90.9 & 95.4 & 90.1 & 93.1 & 93.8 & 90.4 & 97.9 & 93.4 & 93.6 & 90.4 & & \\
\hline Eubacterium formicigenerans & 83.8 & 80.3 & 82.6 & 81.3 & 82.5 & 82.8 & 83.6 & 88.3 & 91.3 & 89.7 & 92.3 & 89.4 & 92.0 & 92.3 & 90.8 & 93.9 & 93.0 & 91.5 & 90.8 & 94.4 & \\
\hline Roseburia cecicola & 83.5 & 80.4 & 83.5 & 82.0 & 83.4 & 83.2 & 83.5 & 87.8 & 92.2 & 90.4 & 92.4 & 90.8 & 94.0 & 92.8 & 93.5 & 92.9 & 93.1 & 93.1 & 90.0 & 93.4 & 91.6 \\
\hline
\end{tabular}




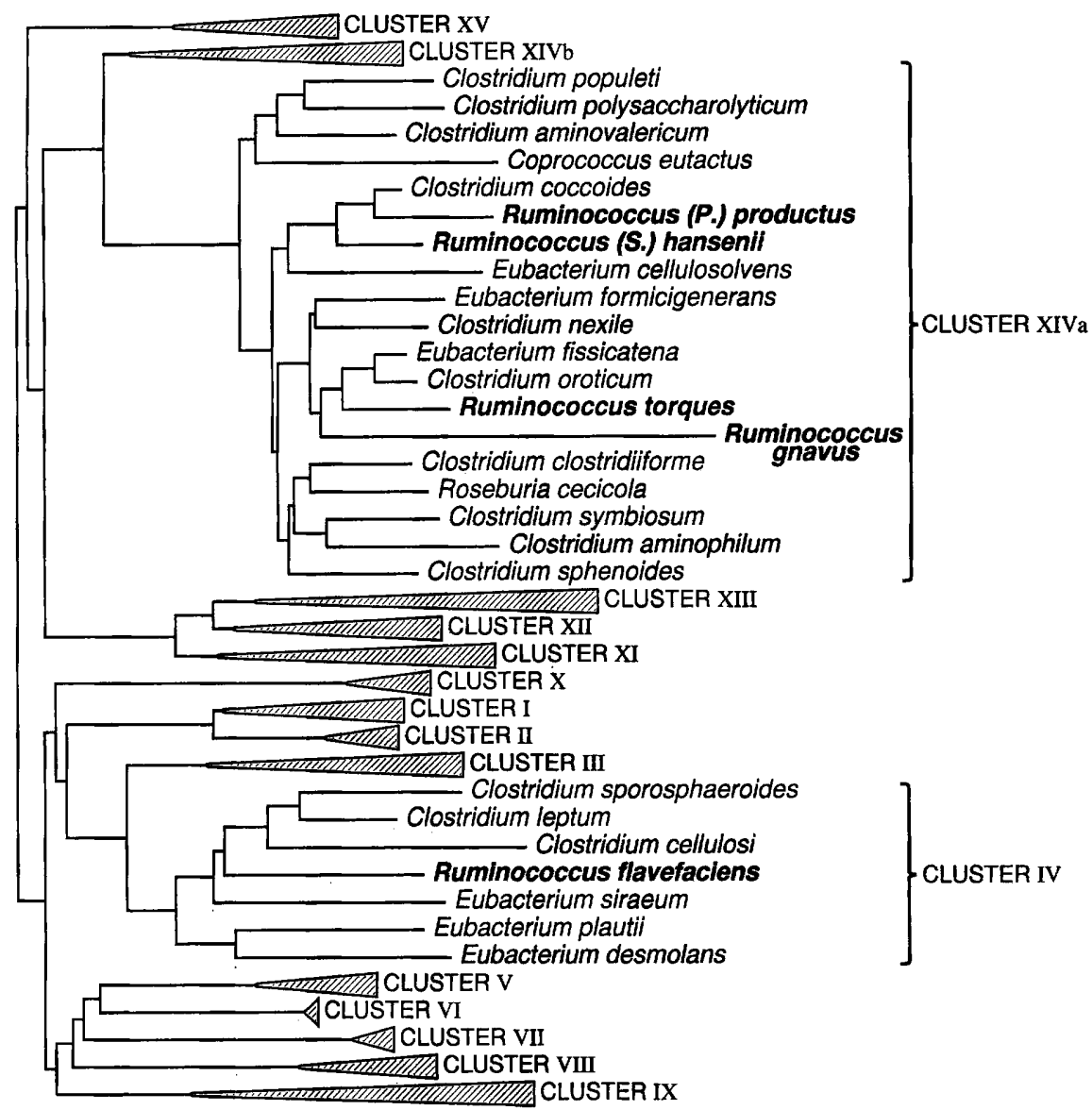

FIG. 1. Unrooted phylogenetic tree showing the position of $R$. flavefaciens within the Clostridium subphylum of the gram-positive bacteria.

imately 85.8 to $88.5 \%$ ) (Table 1) (groups of Collins et al. [2]). $R$. flavefaciens exhibited significantly lower levels of sequence similarity with $R$. gnavus $(79.0 \%), R$. torques $(82.6 \%), R .(P$. productus $(83.1 \%)$, and $R$. $(S$.$) hansenii (83.9 \%)$. We also performed a detailed phylogenetic analysis of $R$. flavefaciens within the Clostridium subphylum. A 1,310-nucleotide region (positions 101 to 1376; Escherichia coli numbering) was aligned by using the program PILEUP (3), and the alignment was corrected manually. A distance matrix was produced by using the program DNADIST of the PHYLIP package (6), and a tree was constructed by the neighbor-joining method with the program NEIGHBOR of the same package. Figure 1 is a tree which shows the position of $R$. flavefaciens within the Clostridium subphylum. Our treeing analysis confirmed that $R$. flavefaciens is closely related to members of Clostridium cluster IV, which includes three clostridial species (Clostridium cellulosi, Clostridium leptum, and Clostridium sporosphaeroides) and three eubacterial species (Eubacterium desmolans, Eubacterium plautii, and Eubacterium siraeum). In contrast, $R$. (P.) productus, $R$. (S.) hansenii, and other ruminococcal species were distantly related and were recovered in Clostridium cluster XIVa (Fig. 1).

It is evident from the comparative sequence analysis that the genus Ruminococcus is not a monophyletic group. $R$. flavefaciens, the type species of the genus, exhibits a distinct relationship with a phenotypically diverse (e.g., sporulating and nonsporulating) group of organisms referred to as Clostridium cluster IV (Fig. 1) (2). In contrast, the other ruminococcal species which we examined are phylogenetically distantly related to $R$. flavefaciens and exhibit a far closer affinity with Clostridium cluster XIVa (2). The latter group contains organisms which exhibit a wide variety of phenotypes and has a level of intracluster sequence divergence of approximately $16.5 \%$, which is more consistent with a suprageneric (i.e., family) association than a single genus. All of cluster XIVa requires extensive taxonomic revision since many of the species are currently classified in the genera Clostridium, Eubacterium, and Ruminococcus (Fig. 1), although they are in fact phylogenetically only remotely related to the type species of their respective genera. Since cluster XIVa contains a great variety of organisms not previously considered to be related, additional comparative phenotypic studies and sequence analyses (e.g., analyses that include other members of the genus Ruminococcus) will be necessary before a taxonomically acceptable subdivision of this cluster can be proposed. However, our data demonstrate that, irrespective of whether $R$. (P.) productus, $R$. $(S$. ) hansenii, $R$. gnavus, and $R$. torques belong to a single genus, it is taxonomically incorrect to preserve the name Ruminococcus for these organisms. Therefore, we suggest that the names Streptococcus hansenii and Peptostreptococcus productus should be retained until the taxonomy and nomenclature of Clostridium cluster XIVa are resolved. As noted above, the species belonging to this cluster are currently assigned to a variety of genera (e.g., the genera Acetitomaculum, Coprococcus, and Roseburia), a fact which will have to be taken into account in 
any future taxonomic revision involving $P$. productus and $S$. hansenii.

Nucleotide sequence accession number. The 1,448-nucleotide sequence which we determined has been deposited in the EMBL data library under accession number X83430.

This work was supported by the Ministry of Agriculture, Fisheries and Food.

\section{REFERENCES}

1. Bryant, M. P. 1986. The genus Ruminococcus Sijpesteijn 1948, p. 1093-1097. In P. H. A. Sneath, N. S. Mair, M. E. Sharpe, and J. G. Holt (ed.), Bergey's manual of systematic bacteriology, vol. 2. The Williams and Wilkins Co., Baltimore.

2. Collins, M. D., P. A. Lawson, A. Willems, J. J. Cordoba, J. FernandezGarayzabal, P. Garcia, J. Cai, H. Hippe, and J. A. E. Farrow. 1994. The phylogeny of the genus Clostridium: proposal of five new genera and eleven new species combinations. Int. J. Syst. Bacteriol. 44:812-826.

3. Devereux, J., P. Haeberli, and O. Smithies. 1984. A comprehensive set of sequence analysis programs for the VAX. Nucleic Acids Res. 12:387-395.

4. Ezaki, T., N. Li, Y. Hashimoto, H. Miura, and H. Yamamoto. 1994. 16S ribosomal DNA sequences of anaerobic cocci and proposal of Ruminococcus hansenii comb. nov. and Ruminococcus productus comb. nov. Int. J. Syst. Bacteriol. 44:130-136.

5. Ezaki, T., H. Oyaizu, and E. Yabuuchi. 1992. The anaerobic Gram-positive cocci, p. 1879-1992. In A. Balows, H. G. Trüper, M. Dworkin, W. Harder, and K.-H. Schleifer (ed.), The prokaryotes. A handbook on the biology of bacteria: ecophysiology, isolation, identification, applications. Springer-Verlag, New York.

6. Felsenstein, J. 1989. PHYLIP-phylogeny inference package (version 3.2). Cladistics 5:164-166.

7. Hardie, J. M. 1986. Other streptococci, p. 1068-1071. In P. H. A. Sneath, N. S. Mair, M. E. Sharpe, and J. G. Holt (ed.), Bergey's manual of systematic bacteriology, vol. 2. The Williams and Wilkins Co., Baltimore.

8. Holdeman Moore, L. V., J. L. Johnson, and W. E. C. Moore. 1986. Genus Peptostreptococcus Kluyver and van Niel 1936, p. 1083-1092. In P. H. A. Sneath, N. S. Mair, M. E. Sharpe, and J. G. Holt (ed.), Bergey's manual of systematic bacteriology, vol. 2. The Williams and Wilkins Co. Baltimore.

9. Hutson, R. A., D. E. Thompson, and M. D. Collins. 1993. Genetic interrelationships of saccharolytic Clostridium botulinum types B, E, and F and related clostridia as revealed by small subunit rRNA sequences. FEMS Microbiol. Lett. 108:103-110.

10. Lawson, P. A., S. E. Gharbia, H. N. Shah, and D. R. Clark. 1989. Recognition of Fusobacterium nucleatum subgroups $\mathrm{Fn}-1, \mathrm{Fn}-2$ and $\mathrm{Fn}-3$ by ribosomal RNA gene restriction patterns. FEMS Microbiol. Lett. 65:41-46.

11. $\mathrm{Li}_{\mathrm{i}}, \mathbf{N}_{\text {., Y }}$. Hashimoto, and T. Ezaki. 1994. Determination of $16 \mathrm{~S}$ ribosomal RNA sequences of all members of the genus Peptostreptococcus and their phylogenetic position. FEMS Microbiol. Lett. 116:1-6.

12. Ludwig, W., M. Weizenegger, R. Kilpper-Bälz, and K. H. Schleifer. 1988. Phylogenetic relationships of anaerobic streptococci. Int. J. Syst. Bacteriol. 38:15-18. 